\title{
Article
}

Mycosphere

Doi 10.5943/mycosphere/7/6/16

Copyright (C) Guizhou Academy of Agricultural Sciences

\section{Multigene phylogeny and HPLC analysis reveal fake Ophiocordyceps sinensis in markets}

Wen $\mathrm{TC}^{1,2}$, Wei DP${ }^{1}$, Long $F Y^{1,2}$, Zeng XY ${ }^{1,3}$, Kang JC ${ }^{1,2 *}$

\author{
1 The Engineering Research Center of Southwest Bio-Pharmaceutical Resources, Ministry of Education, Guizhou \\ University, Guiyang 550025, Guizhou, China \\ ${ }^{2}$ School of Pharmacy, Guizhou University, Guiyang 550025, Guizhou, China \\ ${ }^{3}$ Center of Excellence in Fungal Research, and School of Science, Mae Fah Luang University, Chiang Rai 57100, \\ Thailand
}

Wen TC, Wei DP, Long FY, Zeng XY, Kang JC 2016 - Multigene phylogeny and HPLC analysis reveal fake Ophiocordyceps sinensis in markets. Mycosphere 7(6), 853-867, Doi 10.5943/mycosphere/7/6/16

\begin{abstract}
Ophiocordyceps sinensis (Cordyceps sinensis) has long been a Chinese Traditional Medicine and functional food in China. Because of its valued medicinal effect and improvement in the Chinese economy, market demand for $O$. sinensis has significantly increased in recent years. Here, we use multigene and High Performance Liquid Chromatography (HPLC) analysis of specimens bought from markets to reveal the sale of fake $O$. sinensis in Traditional Chinese Medicine markets. The insect larvae (belonging to Lepidopteran) was a different species to that normally infected by $O$. sinensis. Combined sequence analysis of ITS, nrSSU, EF-1 $\alpha$ and RPB1 gene markers also revealed the putative $O$. sinensis to be Metacordyceps taii. Producers had gone to great lengths to produce remarkably similar fake $O$. sinensis specimens. The insect body was infected with $M$. taii, but the fungus stromata were made from a Ligularia hodgsonii stem that had been molded into stromata and stuck into the insect body. We analyzed the nucleoside constitutes of the insect bodies and fake Cordyceps stromata which were very different to those of authentic wild $O$. sinensis samples from Tibet. It is not clear what the consumers of these products are actually ingesting and whether it may be harmful. In the future, sequence data should be used to test the authenticity of $O$. sinensis, and the development a real-time PCR assay for species-specific diagnosis is needed. The use of multi-gene phylogeny could have a wide application in verification of other Traditional Chinese Medicined and fungal biotechnology products.
\end{abstract}

Key words - Chemical analysis - Combined sequence analysis - fake Cordyceps - Ligularia hodgsonii-Metacordyceps taii

\section{Introduction}

Ophiocordyceps sinensis (syn. Cordyceps sinensis) has long been used as a Traditional Chinese Medicine (TCM) and a tonic in China (Zhou et al. 2009). Because of its revered medicinal effect, and economic improvements in China, the market demand for $O$. sinensis has increased significantly. The price of $O$. sinensis per gram is now higher than that of gold, with best quality samples being 100-130 USD/g in 2012 (Shrestha \& Bawa 2013). This is the result of increased 
demand and over gathering, the latter which has caused substantial reductions in populations (Mortimer et al. 2012).

Ophiocordyceps sinensis, which is gathered from the wild, is a rich source in bioactive compounds (De Silva et al. 2013), such as polysaccharides ( $\mathrm{Li}$ et al. 2006), adenosine and other nucleotide/nucleotides derivatives ( $\mathrm{Li}$ et al. 2004a, 2006), ergosterol (Li et al. 2004a, 2006), $\delta$-3ergosterol (Zhu et al. 1998), cordycepic acid (mannitol) (Li et al. 2006), crude protein, amino acids, and metal elements (Zhu et al. 1998). They manifest a wide range of pharmacological functions (Li et al. 2002, De Silva et al. 2013), and its immunity regulation function plays an important role in antitumor and anticancer activity (De Silva et al. 2012a), prevention and control of diabetes (De Silva et al. 2012b), organ transplants and therapy for some diseases of the kidney, liver and heart (Kuo et al. 1996, Zhou et al. 2009).

Ophiocordyceps sinensis is restricted to the high altitudes of the Himalayas and Tibetan Plateau (Shrestha \& Bawa 2013). It has been collected at altitudes of about 3200-4200 $\mathrm{m}$ in India (Singh et al. 2010), 3540-5050 m in Nepal (Devkota 2000), 4200-5200 m in Bhutan (Cannon et al. 2009), and 3000-5000 $\mathrm{m}$ in Tibet, Qinghai, Sichuan, Yunnan and Gansu Provinces in China (Zhou et al. 2009). Winkler (2009) analyzed available production data and estimated that the total annual production was in the range of 85 to 185 tons for all production areas in the Himalayas and Tibetan plateau. This yields a huge total estimated global market value of US \$5-11 billion (Shrestha 2012). The market price climbed more than nine times in Tibet from 1997 to 2008 (Winkler 2009), and as a result collectors are extending their collecting areas year by year. This has resulted in over exploitation of the pristine landscapes with significant ecosystem degradation (Shrestha 2012).

Methods for the artificial cultivation of $O$. sinensis have not yet been successfully developed, despite considerable funds being committed for this by the Chinese government over the past 30 years (Zhou et al. 2009). Some fermentation products have been produced using the anamorph state of $O$. sinensis ( $\mathrm{Li}$ et al. 1999, 2006, Jiang \& Yao 2003). With high increase in the price of wild $O$. sinensis and a decrease in the annual collection, fake $O$. sinensis have started to emerge in TCM markets. This is because it is difficult for the average customer to identify fake $O$. sinensis. An X-ray machine was developed to reveal the fraudulent practice of placing copper and other metals in the $O$. sinensis insect host which increased the sale value though increased weight (Tuli et al. 2013). However, this cannot detect fake $O$. sinensis, comprising other species of Cordyceps sensu lato, sold in the market.

The purpose of this study was to establish weather fake $O$. sinensis specimens were being sold in TCM markets. In this study, we used analysis of combined ITS, nrSSU, EF-1 $\alpha$, RPB1 sequenced data and HPLC detection of nucleosides to establish weather the $O$. sinensis samples were authentic.

\section{Materials \& Methods}

\section{Specimens and host}

Ophiocordyceps sinensis specimens were collected in Linzhi and Basu County, Tibet, China in May 2010. Specimens of $O$. sinensis were also bought from a Traditional Chinese Medicine market in October 2012. Specimens were stored in plastic containers and transported to the laboratory for identification and analysis.

\section{Morphological characterization}

Material was examined under an Optec SZ660 stereo microscope (Chongqing Optec Instrument Co., Ltd, Chongqing, China) where photographs were taken.

DNA extraction, PCR amplification and determination of DNA sequences

Total genomic DNA of fungal was extracted from dried specimens using an E.Z.N.A. ${ }^{\mathrm{TM}}$ Fungal DNA MiniKit (Omega Biotech Inc., CA, USA) and Plant DNA was extracted from dried specimens using an EZgene ${ }^{\mathrm{TM}}$ Plant gDNA Kit (Biomega Inc., CA, USA) according to manufacturers' protocols and the extracted DNA was stored at -20 C. Two nuclear (ITS, nrSSU) 
and two protein gene (EF-1 $\alpha$, RPB1) loci were amplified and sequenced (Sung et al. 2007), for the undetermined fruiting body of the specimens from market, ITS loci was amplified and sequenced.

The PCR amplification and sequencing of ITS1-5.8S-ITS2 rDNA (ITS) were conducted as described in White et al.(1990), The ITS was amplified and sequenced with the primers ITS4 (5'TCCTCCGCTTATTGATATGC-3') and ITS5 (5'-GGAAGTAAAAGTCGTAACAAGG-3') (White et al. 1990), while primers ITS1 (5'-TCCGTAGGTGAACCTGCGG-3') and ITS4 (5'TCCTCCGCTTATTGATATGC-3') were used when amplifying the undetermined fruiting body (White et al. 1990). The PCR amplification and sequencing of nrSSU were conducted as described in Sung et al. (2007). The nrSSU was amplified and sequenced with the primers NS1 (5'GTAGTCATATGCTTGTCTC-3') and NS4 (5'-CTTCCGTCAATTCCTTTAAG-3') (White et al. 1990). In the amplification of EF-1 $\alpha$ and RPB1, we followed Sung et al. (2007) and Castlebury et al. (2004). For the amplification of EF-1 $\alpha$, the primers 983F (5'GCYCCYGGHCAYCGTGAYTTYAT-3') and 2218R (5'- ATGACACCRACRGCRACRGTYTG3') were used (Sung et al. 2007). Sequencing of RPB1 was performed with the same primers used in the amplification. For RPB1, the primers CRPB1A (5'-CAYCCWGGYTTYATCAAGAA-3') and RPB1Cr (5'-CCNGCDATNTCRTTRTCCATRTA-3') were used in PCR amplification and sequencing (Castlebury et al. 2004).

All PCR products were sequenced by GenScript Biotechnology Co. Ltd. (Nanjing, China).

\section{Sequence alignment and phylogenetic analysis}

The taxon information and GenBank accession numbers used in the molecular analysis are listed in Table 1, the plant information are listed in Table 2. The four gene datasets (ITS, nrSSU, EF-1 $\alpha$, RPB1) from the undetermined specimens and natural Ophiocordyceps sinensis from Tibet, plus datasets obtained from GenBank, were aligned using MEGA5.05 Tamura et al. (2011). Alignments were manually adjusted to allow maximum sequence similarity. Gaps were treated as missing data. Unweighted maximum Parsimony (MP) analysis were performed using PAUP* 4.0b10 (Swofford 2002). Trees were inferred using the heuristic search option with TBR branch swapping and 1000 random sequence additions. Maxtrees were 5000, branches of zero length were collapsed and all multiple parsimonious trees were saved. Clade stability of the trees resulting from the parsimony analyses were assessed by bootstrap analysis with 1000 replicates, each with 10 replicates of random stepwise addition of taxa (Felsenstein 1985). Trees were viewed in Treeview and exported to graphics programs (Page 1996).

\section{Nucleosides Analytical methods}

The samples were dried overnight to a constant weight at $55^{\circ} \mathrm{C}$. Cordycepin, adenosine and other nucleosides in fruiting bodies and the host insect was analyzed by HPLC (1200 series, Agilent Technology, U.S.) with a C18 reverse phase column $(5 \mu \mathrm{m}, 4.6 \times 150 \mathrm{~mm}$, Upelco, Bellefonte, PA, USA). Standard cordycepin, adenosine and other nucleoside samples from Sigma were dissolved in distilled water for calibration. The mobile phase was $10 \mathrm{mM} \mathrm{KH}_{2} \mathrm{PO}_{4}$, which was dissolved in methanol/distilled water (6:94). Elution was performed at a flow rate of $1 \mathrm{ml} / \mathrm{min}$ with column temperature at $45^{\circ} \mathrm{C}$ and at a UV wavelength of $259 \mathrm{~nm}$.

\section{Results}

The partition homogeneity test $(P=0.01)$ suggested that the individual gene partitions were not highly incongruent (Farris et al. 1994, Cunningham 1997). Blast searches were made and recent publications studied to reveal the closest matches in GenBank for phylogenetic analysis (Table 1). The combined datasets comprised 3456 characters after alignment, of which 914 characters are parsimony-informative, 2089 constant, and 453 parsimony-uninformative. Parsimony analysis generated 5000 trees; $\mathrm{SH}$ test verified that they were similar, one of which (tree length $=3907$ steps, $\mathrm{CI}=0.530, \mathrm{RI}=0.777, \mathrm{RC}=0.412, \mathrm{HI}=0.470$ ) and the most parsimonies tree is shown in Fig. 2. 
Table 1 Taxa used in molecular phylogenetic analyses in fungals.

\begin{tabular}{|c|c|c|c|c|c|c|c|}
\hline \multirow{2}{*}{ Species } & \multirow{2}{*}{ Voucher Info. ${ }^{1}$} & \multirow{2}{*}{$\begin{array}{l}\text { Host/Substratu } \\
\text { m }\end{array}$} & \multicolumn{4}{|c|}{ GenBank Accession Number } & \multirow{2}{*}{-References } \\
\hline & & & ITS & nrSSU & $\overline{E F-1 \alpha}$ & RPB1 & \\
\hline $\begin{array}{l}\text { Metacordyceps taii } \\
\text { (host) }\end{array}$ & $\begin{array}{l}\text { GZUH } \\
\text { 2012HK2 }\end{array}$ & $\begin{array}{l}\text { Lepidopteran } \\
\text { pupa }\end{array}$ & KJ364485 & KR153587 & KJ364493 & & In this study \\
\hline $\begin{array}{l}\text { Metacordyceps taii } \\
\text { (host) }\end{array}$ & $\begin{array}{l}\text { GZUH } \\
\text { 2012HK6 }\end{array}$ & $\begin{array}{l}\text { Lepidopteran } \\
\text { pupa }\end{array}$ & KJ364486 & & KJ364494 & & In this study \\
\hline $\begin{array}{l}\text { Ophiocordyceps } \\
\text { sinensis }\end{array}$ & GZUH 2010LL4 & $\begin{array}{l}\text { Hepialus } \\
\text { armoricanus }\end{array}$ & KJ364487 & KJ364490 & KJ364495 & KJ364498 & In this study \\
\hline $\begin{array}{l}\text { Ophiocordyceps } \\
\text { sinensis }\end{array}$ & $\begin{array}{l}\text { GZUH } \\
\text { 2010RW4 }\end{array}$ & $\begin{array}{l}\text { Hepialus } \\
\text { armoricanus }\end{array}$ & KJ364488 & KJ364491 & KJ364496 & KJ364499 & In this study \\
\hline $\begin{array}{l}\text { Ophiocordyceps } \\
\text { sinensis }\end{array}$ & $\begin{array}{l}\text { GZUH } \\
\text { 2010RW5 }\end{array}$ & $\begin{array}{l}\text { Hepialus } \\
\text { armoricanus }\end{array}$ & KJ364489 & KJ364492 & KJ364497 & & In this study \\
\hline $\begin{array}{l}\text { Cordyceps } \\
\text { brongniartii }\end{array}$ & NBRC 101395 & & JN943298 & JN941759 & & JN992493 & Schoch et al. 2012 \\
\hline $\begin{array}{l}\text { Cordyceps } \\
\text { brongniartii }\end{array}$ & BCC 16585 & & JN049867 & JF415951 & JF416009 & JN049885 & Kepler et al. 2012 \\
\hline $\begin{array}{l}\text { Cordyceps } \\
\text { cardinalis }\end{array}$ & CBS $113411 *$ & $\begin{array}{l}\text { Lepidopteran } \\
\text { larva }\end{array}$ & & AY184973 & DQ522325 & DQ522370 & Sung et al. 2007 \\
\hline $\begin{array}{l}\text { Cordyceps } \\
\text { cardinalis }\end{array}$ & OSC 93610 & & JN049843 & AY184974 & EF469059 & EF469088 & Kepler et al. 2012 \\
\hline Cordyceps militaris & OSC 93623 & $\begin{array}{l}\text { Lepidopteran } \\
\text { pupa }\end{array}$ & JN049825 & AY184977 & DQ522332 & DQ522377 & Sung et al. 2007 \\
\hline Cordyceps militaris & NBRC 100741 & & JN943437 & JN941755 & & JN992489 & Schoch et al. 2012 \\
\hline $\begin{array}{l}\text { Elaphocordyceps } \\
\text { japonica }\end{array}$ & OSC 110991 & $\begin{array}{l}\text { Elaphomyces sp. } \\
\text { (Eurotiomycetes) }\end{array}$ & JN049824 & DQ522547 & DQ522330 & DQ522375 & Sung et al. 2007 \\
\hline $\begin{array}{l}\text { Elaphocordyceps } \\
\text { japonica }\end{array}$ & IFO 9647 & & $\begin{array}{l}\mathrm{AB} 02736 \\
6\end{array}$ & $\mathrm{AB} 027320$ & & & $\begin{array}{l}\text { Nikoh \& Fukatsu } \\
2000\end{array}$ \\
\hline $\begin{array}{l}\text { Elaphocordyceps } \\
\text { ophioglossoides }\end{array}$ & OSC 106405 & $\begin{array}{l}\text { Elaphomyces sp. } \\
\text { (Eurotiomycetes) }\end{array}$ & & AY489691 & AY489618 & AY489652 & Sung et al. 2007 \\
\hline $\begin{array}{l}\text { Elaphocordyceps } \\
\text { ophioglossoides }\end{array}$ & NBRC 106331 & & JN943320 & JN941733 & & JN992467 & Schoch et al. 2012 \\
\hline $\begin{array}{l}\text { Elaphocordyceps } \\
\text { subsessilis }\end{array}$ & OSC 71235 & $\begin{array}{l}\text { Scarabaeid larva } \\
\text { (Coleoptera) }\end{array}$ & JN049844 & EF469124 & EF469061 & EF469090 & Sung et al. 2007 \\
\hline $\begin{array}{l}\text { Metacordyceps } \\
\text { atrovirens }\end{array}$ & TNM-F 10184 & Coleoptera & JN049882 & JF415950 & & JN049884 & Kepler et al. 2012 \\
\hline $\begin{array}{l}\text { Metacordyceps } \\
\text { brittlebankisoides }\end{array}$ & G97025* & & AJ309332 & & & & Liu et al. 2002 \\
\hline $\begin{array}{l}\text { Metacordyceps } \\
\text { campsosterni }\end{array}$ & HMIGD 20885* & & $\begin{array}{l}\text { DQ15024 } \\
7\end{array}$ & & & & Zhang 2005 \\
\hline $\begin{array}{l}\text { Metacordyceps } \\
\text { campsosterni }\end{array}$ & HMIGD 20884 & & $\begin{array}{l}\text { DQ15024 } \\
6\end{array}$ & & & & Zhang 2005 \\
\hline $\begin{array}{l}\text { Metacordyceps } \\
\text { chlamydosporia }\end{array}$ & CBS 101244 & & JN049821 & DQ522544 & DQ522327 & DQ522372 & Kepler et al. 2012 \\
\hline $\begin{array}{l}\text { Metacordyceps } \\
\text { chlamydosporia }\end{array}$ & CBS 504.66 & Nematode & AJ292398 & AF339593 & EF469069 & EF469098 & Sung et al. 2012 \\
\hline $\begin{array}{l}\text { Metacordyceps } \\
\text { guniujiangensis }\end{array}$ & GNJ020527-04* & & $\begin{array}{l}\text { AY91375 } \\
7\end{array}$ & & & & Li et al. 2010 \\
\hline $\begin{array}{l}\text { Metacordyceps } \\
\text { indigotica }\end{array}$ & TNS-F18553 & Lepidoptera & JN049874 & JF415952 & JF416010 & JN049886 & Kepler et al. 2012 \\
\hline $\begin{array}{l}\text { Metacordyceps } \\
\text { indigotica }\end{array}$ & TNS-F18554 & Lepidoptera & JN049875 & JF415953 & JF416011 & JN049887 & Kepler et al. 2012 \\
\hline $\begin{array}{l}\text { Metacordyceps } \\
\text { khaoyaiensis }\end{array}$ & BCC 12687 & Lepidoptera & JN049868 & & JF416013 & JN049889 & Kepler et al. 2012 \\
\hline $\begin{array}{l}\text { Metacordyceps } \\
\text { khaoyaiensis }\end{array}$ & BCC 14290 & Lepidoptera & JN049869 & & JF416012 & JN049888 & Kepler et al. 2012 \\
\hline $\begin{array}{l}\text { Metacordyceps } \\
\text { kusanagiensis }\end{array}$ & TNS F18494 & Coleoptera & JN049873 & JF415954 & JF416014 & JN049890 & Kepler et al. 2012 \\
\hline
\end{tabular}




\begin{tabular}{|c|c|c|c|c|c|c|c|}
\hline $\begin{array}{l}\text { Metacordyceps } \\
\text { liangshanensis }\end{array}$ & EFCC 1452 & $\begin{array}{l}\text { Lepidopteran } \\
\text { pupa }\end{array}$ & & EF468962 & EF468756 & & Sung et al. 2007 \\
\hline $\begin{array}{l}\text { Metacordyceps } \\
\text { liangshanensis }\end{array}$ & EFCC 1523 & $\begin{array}{l}\text { Lepidopteran } \\
\text { pupa }\end{array}$ & & EF468961 & EF468755 & & Sung et al. 2007 \\
\hline $\begin{array}{l}\text { Metacordyceps } \\
\text { martialis }\end{array}$ & TTZ070716-04 & Lepidoptera & JN049871 & JF415955 & & JN049891 & Kepler et al. 2012 \\
\hline $\begin{array}{l}\text { Metacordyceps } \\
\text { martialis }\end{array}$ & $\begin{array}{l}\text { HMAS } \\
197472(\mathrm{~S})\end{array}$ & Lepidoptera & JN049881 & JF415956 & JF416016 & JN049892 & Kepler et al. 2012 \\
\hline $\begin{array}{l}\text { Metacordyceps } \\
\text { owariensis }\end{array}$ & NBRC 33258 & Hemiptera & JN049883 & & JF416017 & & Kepler et al. 2012 \\
\hline $\begin{array}{l}\text { Metacordyceps } \\
\text { pseudoatrovirens }\end{array}$ & TNSF 16380 & Coleoptera & JN049870 & & & JN049893 & Kepler et al. 2012 \\
\hline Metacordyceps taii & ARSEF 5714 & Lepidoptera & JN049829 & AF543763 & AF543775 & DQ522383 & Sung et al. 2007 \\
\hline $\begin{array}{l}\text { Metacordyceps } \\
\text { yongmunensis }\end{array}$ & EFCC 2131 & Lepidoptera & JN049856 & EF468977 & EF468770 & EF468876 & Kepler et al. 2012 \\
\hline $\begin{array}{l}\text { Metacordyceps } \\
\text { yongmunensis }\end{array}$ & EFCC 2135 & Lepidoptera & & EF468979 & EF468769 & EF468877 & Kepler et al. 2012 \\
\hline $\begin{array}{l}\text { Metarhizium } \\
\text { acridum }\end{array}$ & ARSEF 324 & Orthoptera & $\begin{array}{l}\text { HQ33145 } \\
7\end{array}$ & & EU248844 & EU248896 & $\begin{array}{l}\text { Schneider et al. } \\
2011 \text { \& Bischoff } \\
\text { et al. } 2009\end{array}$ \\
\hline $\begin{array}{l}\text { Metarhizium } \\
\text { acridum }\end{array}$ & ARSEF 7486* & Orthoptera & $\begin{array}{l}\text { HQ33145 } \\
8\end{array}$ & & EU248845 & EU248897 & $\begin{array}{l}\text { Schneider et al. } \\
2011 \text { \& Bischoff } \\
\text { et al. } 2009\end{array}$ \\
\hline $\begin{array}{l}\text { Metarhizium } \\
\text { anisopliae }\end{array}$ & ARSEF 7450 & Coleoptera & $\begin{array}{l}\text { HQ33146 } \\
4\end{array}$ & & EU248852 & EU248904 & $\begin{array}{l}\text { Schneider et al. } \\
2011 \& \text { Bischoff } \\
\text { et al. } 2009\end{array}$ \\
\hline $\begin{array}{l}\text { Metarhizium } \\
\text { anisopliae }\end{array}$ & ARSEF 7487* & Orthoptera & $\begin{array}{l}\text { HQ33144 } \\
6\end{array}$ & & DQ463996 & DQ468355 & $\begin{array}{l}\text { Schneider et al. } \\
2011 \text { \& Bischoff } \\
\text { et al. } 2009\end{array}$ \\
\hline $\begin{array}{l}\text { Metarhizium } \\
\text { brunneum }\end{array}$ & ARSEF 2107\{ & Coleoptera & $\begin{array}{l}\text { KC17869 } \\
1\end{array}$ & & EU248855 & EU248907 & $\begin{array}{l}\text { Kepler et al. } 2013 \\
\text { \& Bischoff et al. } \\
2009\end{array}$ \\
\hline $\begin{array}{l}\text { Metarhizium } \\
\text { brunneum }\end{array}$ & ARSEF 4152 & Soil & $\begin{array}{l}\text { HQ33145 } \\
2\end{array}$ & & EU248853 & EU248905 & $\begin{array}{l}\text { Schneider et al. } \\
2011 \& \text { Bischoff } \\
\text { et al. } 2009\end{array}$ \\
\hline $\begin{array}{l}\text { Metarhizium } \\
\text { flavoviride }\end{array}$ & ARSEF $2133^{*}$ & Coleoptera & & & DQ463999 & DQ468358 & $\begin{array}{l}\text { Bischoff et al. } \\
2009\end{array}$ \\
\hline $\begin{array}{l}\text { Metarhizium } \\
\text { frigidum }\end{array}$ & ARSEF 4124* & Coleoptera & & & DQ464002 & DQ468361 & $\begin{array}{l}\text { Bischoff et al. } \\
2009\end{array}$ \\
\hline $\begin{array}{l}\text { Metarhizium } \\
\text { globosum }\end{array}$ & ARSEF 2596* & Lepidoptera & $\begin{array}{l}\text { HQ33145 } \\
9\end{array}$ & & EU248846 & EU248898 & $\begin{array}{l}\text { Schneider et al. } \\
2011 \& \text { Bischoff } \\
\text { et al. } 2009\end{array}$ \\
\hline $\begin{array}{l}\text { Metarhizium } \\
\text { guizhouense }\end{array}$ & CBS 258.90* & Lepidoptera & $\begin{array}{l}\text { HQ33144 } \\
8\end{array}$ & & EU248862 & EU248914 & $\begin{array}{l}\text { Schneider et al. } \\
2011 \text { \& Bischoff } \\
\text { et al. } 2009\end{array}$ \\
\hline $\begin{array}{l}\text { Metarhizium } \\
\text { guizhouense }\end{array}$ & ARSEF 6238 & Lepidoptera & $\begin{array}{l}\text { HQ33144 } \\
7\end{array}$ & & EU248857 & EU248909 & $\begin{array}{l}\text { Schneider et al. } \\
2011 \text { \& Bischoff } \\
\text { et al. } 2009\end{array}$ \\
\hline $\begin{array}{l}\text { Metarhizium } \\
\text { lepidiotae }\end{array}$ & ARSEF 7412 & Coleoptera & $\begin{array}{l}\text { HQ33145 } \\
5\end{array}$ & & EU248864 & EU248916 & $\begin{array}{l}\text { Schneider et al. } \\
2011 \& \text { Bischoff } \\
\text { et al. } 2009\end{array}$ \\
\hline $\begin{array}{l}\text { Metarhizium } \\
\text { lepidiotae }\end{array}$ & ARSEF 7488* & Coleoptera & $\begin{array}{l}\text { HQ33145 } \\
6\end{array}$ & & EU248865 & EU248917 & $\begin{array}{l}\text { Schneider et al. } \\
2011 \text { \& Bischoff } \\
\text { et al. } 2009\end{array}$ \\
\hline Metarhizium majus & ARSEF 1015 & Lepidoptera & $\begin{array}{l}\text { HQ33144 } \\
4\end{array}$ & & EU248866 & EU248918 & $\begin{array}{l}\text { Schneider et al. } \\
2011 \& \text { Bischoff } \\
\text { et al. } 2009\end{array}$ \\
\hline Metarhizium majus & ARSEF 1914\{ & Coleoptera & $\begin{array}{l}\text { HQ33144 } \\
5\end{array}$ & & EU248868 & EU248920 & $\begin{array}{l}\text { Schneider et al. } \\
2011 \& \text { Bischoff } \\
\text { et al. } 2009\end{array}$ \\
\hline $\begin{array}{l}\text { Metarhizium } \\
\text { pingshaense }\end{array}$ & ARSEF 3210 & Isoptera & $\begin{array}{l}\text { HQ33144 } \\
9\end{array}$ & & DQ463995 & DQ468354 & $\begin{array}{l}\text { Schneider et al. } \\
2011 \text { \& Bischoff }\end{array}$ \\
\hline
\end{tabular}




\begin{tabular}{|c|c|c|c|c|c|c|c|}
\hline $\begin{array}{l}\text { Metarhizium } \\
\text { pingshaense }\end{array}$ & CBS 257.90* & Coleoptera & $\begin{array}{l}\text { HQ33145 } \\
0\end{array}$ & & EU248850 & EU248902 & $\begin{array}{l}\text { Schneider et al. } \\
2011 \text { \& Bischoff } \\
\text { et al. } 2009\end{array}$ \\
\hline $\begin{array}{l}\text { Metarhizium } \\
\text { robertsii }\end{array}$ & ARSEF 727 & Orthoptera & $\begin{array}{l}\text { HQ33145 } \\
3\end{array}$ & & DQ463994 & DQ468353 & $\begin{array}{l}\text { Schneider et al. } \\
2011 \text { \& Bischoff } \\
\text { et al. } 2009\end{array}$ \\
\hline $\begin{array}{l}\text { Metarhizium } \\
\text { robertsii }\end{array}$ & ARSEF 7501 & & & & EU248849 & EU248901 & $\begin{array}{l}\text { Bischoff et al. } \\
2009\end{array}$ \\
\hline $\begin{array}{l}\text { Ophiocordyceps } \\
\text { rhizoidea }\end{array}$ & N.H.J. 12522 & $\begin{array}{l}\text { Termite } \\
\text { (Isoptera) }\end{array}$ & JN049857 & EF468970 & EF468764 & EF468873 & Sung et al. 2007 \\
\hline $\begin{array}{l}\text { Ophiocordyceps } \\
\text { rhizoidea }\end{array}$ & N.H.J. 12529 & $\begin{array}{l}\text { Termite } \\
\text { (Isoptera) }\end{array}$ & & EF468969 & EF468765 & EF468872 & Sung et al. 2007 \\
\hline $\begin{array}{l}\text { Ophiocordyceps } \\
\text { sinensis }\end{array}$ & EFCC 7287 & $\begin{array}{l}\text { Lepidopteran } \\
\text { pupa }\end{array}$ & JN049854 & EF468971 & EF468767 & EF468874 & Sung et al. 2007 \\
\hline $\begin{array}{l}\text { Ophiocordyceps } \\
\text { sinensis }\end{array}$ & ARSEF 6282 & & $\begin{array}{l}\text { HM59598 } \\
1\end{array}$ & & HM595918 & HM595952 & Chan et al. 2011 \\
\hline $\begin{array}{l}\text { Ophiocordyceps } \\
\text { stylophora }\end{array}$ & OSC 111000 & $\begin{array}{l}\text { Elaterid larva } \\
\text { (Coleoptera) }\end{array}$ & JN049828 & DQ522552 & DQ522337 & DQ522382 & Sung et al. 2007 \\
\hline $\begin{array}{l}\text { Ophiocordyceps } \\
\text { stylophora }\end{array}$ & OSC 110999 & $\begin{array}{l}\text { Coleopteran } \\
\text { larva }\end{array}$ & & EF468982 & EF468777 & EF468882 & Sung et al. 2007 \\
\hline $\begin{array}{l}\text { Ophiocordyceps } \\
\text { yakusimensis }\end{array}$ & & $\begin{array}{l}\text { Nymph of cicada } \\
\text { (Hemiptera) }\end{array}$ & $\begin{array}{l}\mathrm{AB} 04464 \\
3\end{array}$ & AB044632 & & & $\begin{array}{l}\text { Nikoh \& Fukatsu } \\
2000\end{array}$ \\
\hline $\begin{array}{l}\text { Tyrannicordyceps } \\
\text { fratricida }\end{array}$ & TNS 19011* & Fungi & JQ349068 & JQ257022 & JQ257028 & JQ257016 & Kepler et al. 2012 \\
\hline $\begin{array}{l}\text { Glomerella } \\
\text { cingulata }\end{array}$ & CBS 114054 & $\begin{array}{l}\text { Fragaria sp. } \\
\text { (Rosaceae) }\end{array}$ & $\begin{array}{l}\text { DQ28620 } \\
2\end{array}$ & AF543762 & & AY489659 & Sung et al. 2007 \\
\hline
\end{tabular}

1 A.E.G., A. E. Glenn personal collection; ARSEF, USDA-ARS Collection of Entomopathogenic Fungal cultures, Ithaca, NY; ATCC, American Type Culture Collections, Manassas, VA; BCC, BIOTEC Culture Collection, Klong Luang, Thailand; CBS, Centraalbureau voor Schimmelcultures, Utrecht, the Netherlands; EFCC, Entomopathogenic Fungal Culture Collection, Chuncheon, Korea; F.A.U., F. A. Uecker personal collection; E.G.S., E, G. Simmons personal collection; GAM, Julian H. Miller Mycological Herbarium Athens, GA; G.J.S., G. J. Samuels personal collection; GZUH, Guizhou University Herbarium, Guiyang, Guizhou, China; KEW, mycology collection of Royal Botanical Garden, KEW, Surrey, UK; MICH, University of Michigan Herbarium, Ann Arbor, MI; N.H.J., Nigel Hywel-Jones personal collection; OSC, Oregon State University Herbarium, Corvallis, OR; S.A., S. Alderman personal collection.

* Denotes an ex-type isolate. \{ Denotes an ex-epitype isolate.

The data set comprises 36 species including three Cordyceps, three Ophiocordyceps, three Elaphocordyceps, and 27 Metacordyceps/Metarhizium species. The samples sold in a TCM market as Ophiocordyceps sinensis clustered with M. taii in the Metacordyceps-Metarhizium clade with relatively high bootstrap support (97\%). The Ophiocordyceps sinensis samples from Tibet clustered with $O$. sinensis in the Ophiocordyceps clade with high bootstrap support (100\%).

The host insect of the fake specimens is not Hepialus armoricanus, and the fungus is not Ophiocordyceps sinensis, but is Metacordyceps taii (asexual state = Metarhizium guizhouense). In the fake samples the insect was identified as a lepidopteran larvae and these were infected by Metacordyceps taii. However, the fungus stromata was in fact false and was the stem of a plant (Fig. 1). Phylogenetic analysis of ITS gene of the genus Ligularia has proved by $100 \%$ bootstrap that this stem belongs to the clade of Ligularia hodgsonii (Fig. 4). This had been stuck to the insect body and its resemblance to authentic Ophiocordyceps sinensis specimens was remarkable. When chemically tested, the false fungal stromata contained only nine nucleosides, and lacked uracil, adenine, and guanosine which are found in natural $O$. sinensis stromata. We also analyzed the nucleosides of the insect bodies from the fake ones which were different from the wild $O$. sinensis from Tibet (Fig. 3). The insect body of wild $O$. sinensis contains 13 nucleosides, while the fake ones contained 12 nucleosides. 


\section{Discussion}

Ophiocordyceps sinensis is one of three Cordyceps sensu lato species listed in Chinese pharmacopoeia (The State Pharmacopoeia Committee of People Republic of China 2010) and is highly valued in TCM attracting a price of 100-130 USD/g per gram, which is higher than that of gold (Shrestha \& Bawa 2013). Thus the practice of producing fake Ophiocordyceps sinensis is likely to become more common and elaborate methods are likely to be developed to produce specimens to sell in the TCM markets. Previous studies have found that nucleosides are the most important bioactive constituents in $O$. sinensis (Tuli et al. 2013, Yue et al. 2013) and thus the determination of nucleosides in $O$. sinensis is very important for its quality control (Zhu et al. 1998). Winkler (2009) estimated that the total annual production of $O$. sinensis was in the range of 85 to 185 tons for all production areas in the Himalayas and Tibetan plateau, but the total amount sold in markets is over 200 tons per year all over the world. One fraudulent practice of placing copper, mercury and other materials in the $O$. sinensis insect host body was developed to increase the weight to increase profits ( $\mathrm{Wu}$ et al. 1996). This creates a health risk for patients who take the medicine, and there have been several reported clinical poisoning cases (Wu et al. 1996).

Table 2 Taxa used in molecular phylogenetic analyses in fake fruiting bodies.

\begin{tabular}{|c|c|c|}
\hline Species & $\begin{array}{c}\text { GenBank Accession No. } \\
\text { (ITS) }\end{array}$ & References \\
\hline Ligularia hodgsonii (GZUH 2012HK2) & KR153586 & In this study \\
\hline Ligularia anoleuca & AB557884 & Nagano et al. 2010 \\
\hline Ligularia brassicoides & AY723266 & Liu et al. 2006 \\
\hline Ligularia cymbulifera & JF976813 & Li et al. 2011 \\
\hline Ligularia dentata & AY723256 & Li et al. 2011 \\
\hline Ligularia dictyoneura & JF976815 & Li et al. 2011 \\
\hline Ligularia fischeri & AB369647 & Nomura et al. 2010 \\
\hline Ligularia lamarum & AB426703 & Saito et al. 2011 \\
\hline Ligularia lankongensis & AB684267 & Hirota et al. 2012 \\
\hline Ligularia liatroides & AY723268 & Liu et al. 2006 \\
\hline Ligularia lingiana & JF767246 & Yu et al. 2011 \\
\hline Ligularia nelumbifolia & JF976821 & Li et al. 2011 \\
\hline Ligularia pleurocaulis & JF976823 & Li et al. 2011 \\
\hline Ligularia przewalskii & AY723263 & Liu et al. 2006 \\
\hline Ligularia pubifolia & GU444022 & Lei et al. 2011 \\
\hline Ligularia purdomii & AY723257 & Liu et al. 2006 \\
\hline Ligularia sagitta & AY723265 & Liu et al. 2006 \\
\hline Ligularia stenocephala & AB369649 & Nomura et al. 2010 \\
\hline Ligularia subspicata & JF976829 & Li et al. 2011 \\
\hline Ligularia tongolensis & JF976833 & Li et al. 2011 \\
\hline Ligularia tsangschanensis & AY723264 & Liu et al. 2006 \\
\hline Ligularia veitchiana & AB557886 & Nagano et al. 2010 \\
\hline Ligularia vellerea & JF976836 & Li et al. 2011 \\
\hline Ligularia virgaurea & JF976840 & Li et al. 2011 \\
\hline Tussilago farfara & EU785941 & GenBank \\
\hline Ligularia hodgsonii & FJ980336 & GenBank \\
\hline Ligularia hodgsonii & JX402629 & Xiang et al. 2013 \\
\hline Ligularia hodgsonii & KF003090 & Xiang et al. 2013 \\
\hline
\end{tabular}



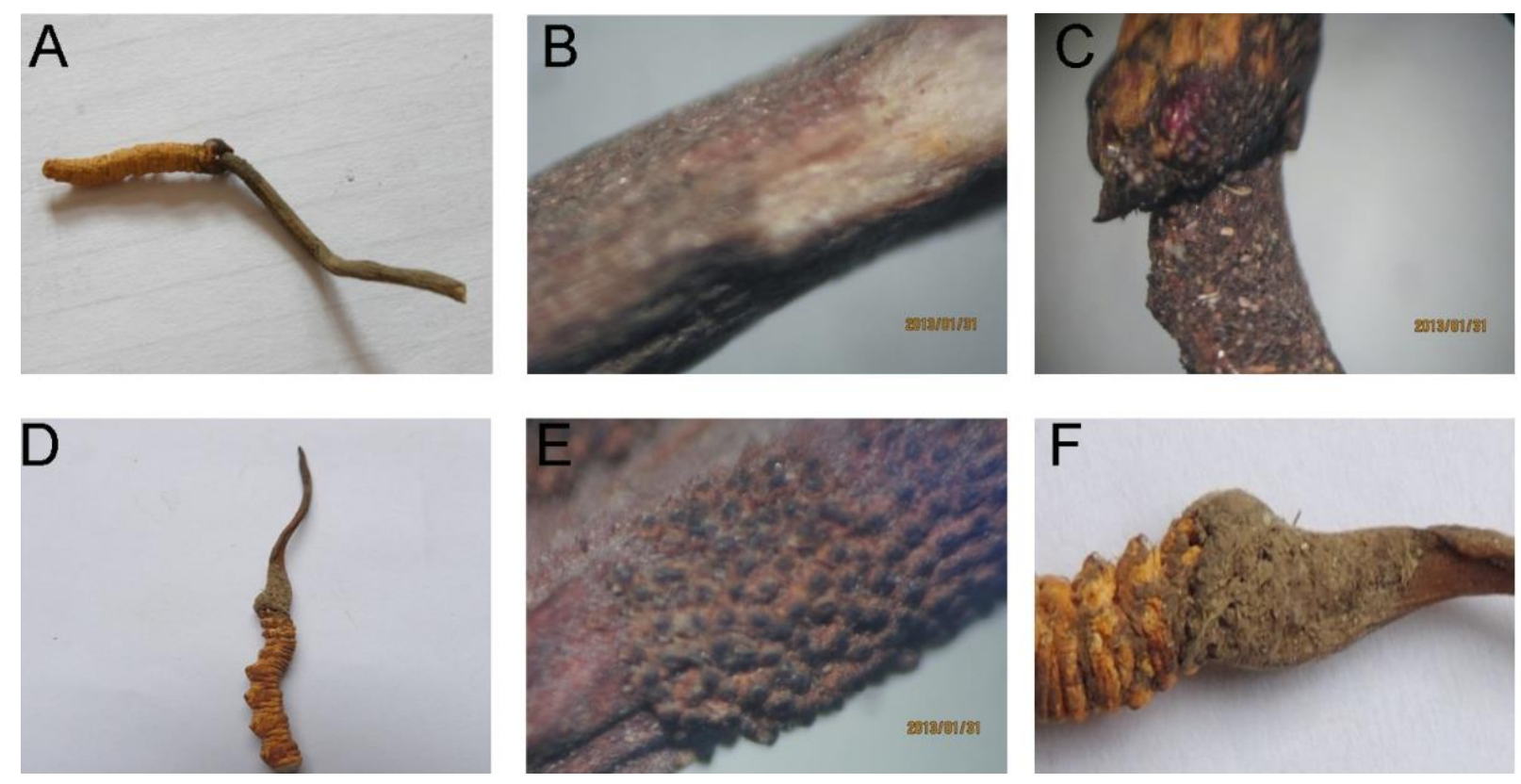

Fig. 1 - Fake (A-C) and wild (D-F) Ophiocordyceps sinensis. A. Fake Ophiocordyceps sinensis showing dead insect and false stroma. Note how the fungal stroma appears to be stuck in the insect. B. Fake stromata with surface sliced (white area) to reveal inner part. There are no ascomata. C. Join between fake stromata and insect larvae. It appears to have been stuck inside the insect. D. Wild Ophiocordyceps sinensis with insect and fungus stroma. E. Dark brown, nearly superficial ascomata on stromata of wild Ophiocordyceps sinensis. F. Area where fungus emerges from insect larvae.

The need to develop methods to detect fake $O$. sinensis is paramount. In China, adenosine is presently the only chemical constituent tested to authenticate and control the quality of $O$. sinensis (The State Pharmacopoeia Committee of People Republic of China 2010). X-ray machines however, were developed to reveal the fraudulent practice of placing copper and other metals in the $O$. sinensis insect host (Tuli et al. 2013). Au et al. (2012) used light and polarized microscopy to compare the cell tissues of the insect and stromata to authenticate $O$. sinensis artificial counterfeits and some fermented Cordyceps products as well as Cordyceps capsules available in markets. They showed that microscopy is a reliable method that needs only few samples for the authentication of Ophiocordyceps sinensis and its related products. Markers and distinct fingerprints from different constituents from sources of Cordyceps have also been reported (Gong et al. 2004, Li et al. 2004b). Several analytical techniques are available to detect of the main nucleosides in Ophiocordyceps sinensis, such as thin layer chromatography (TLC) (Hu et al. 2008, Ma et al. 2008), and capillary electrophoresis (Li et al. 2008, Sun et al. 2008). However, these methods can only determine one or several nucleosides with relatively high content in $O$. sinensis, and trace amounts of several other nucleosides or its derivatives cannot be detected by the above two methods. This is not accurate for distinguishing the more than 400 species of the Cordyceps sensu lato (Wen et al. 2013) and counterfeit production of Ophiocordyceps sinensis and may be why the counterfeiters added nucleotides to the false stromata. In this study, 13 nucleosides and derivatives were detected from natural $O$. sinensis in a single experimental run with high performance liquid chromatography (HPLC). This method can therefore be used for effective quality control of $O$. sinensis.

Some authors have reported that a single gene marker can be used for the identification of O. sinensis and its related products (Hseu \& Chen 2001, Weng \& Li 2008). Unfortunately, these methods are not thoroughly optimized, and extensive work is still needed to accurately identify different Cordyceps sensu lato species and products in the market with high efficiency. No effective molecular diagnosis protocol provides a specific and sensitive probe for this important and expensive TCM. It is necessary to identify a SNP - rich region to enable rapid species-specific 


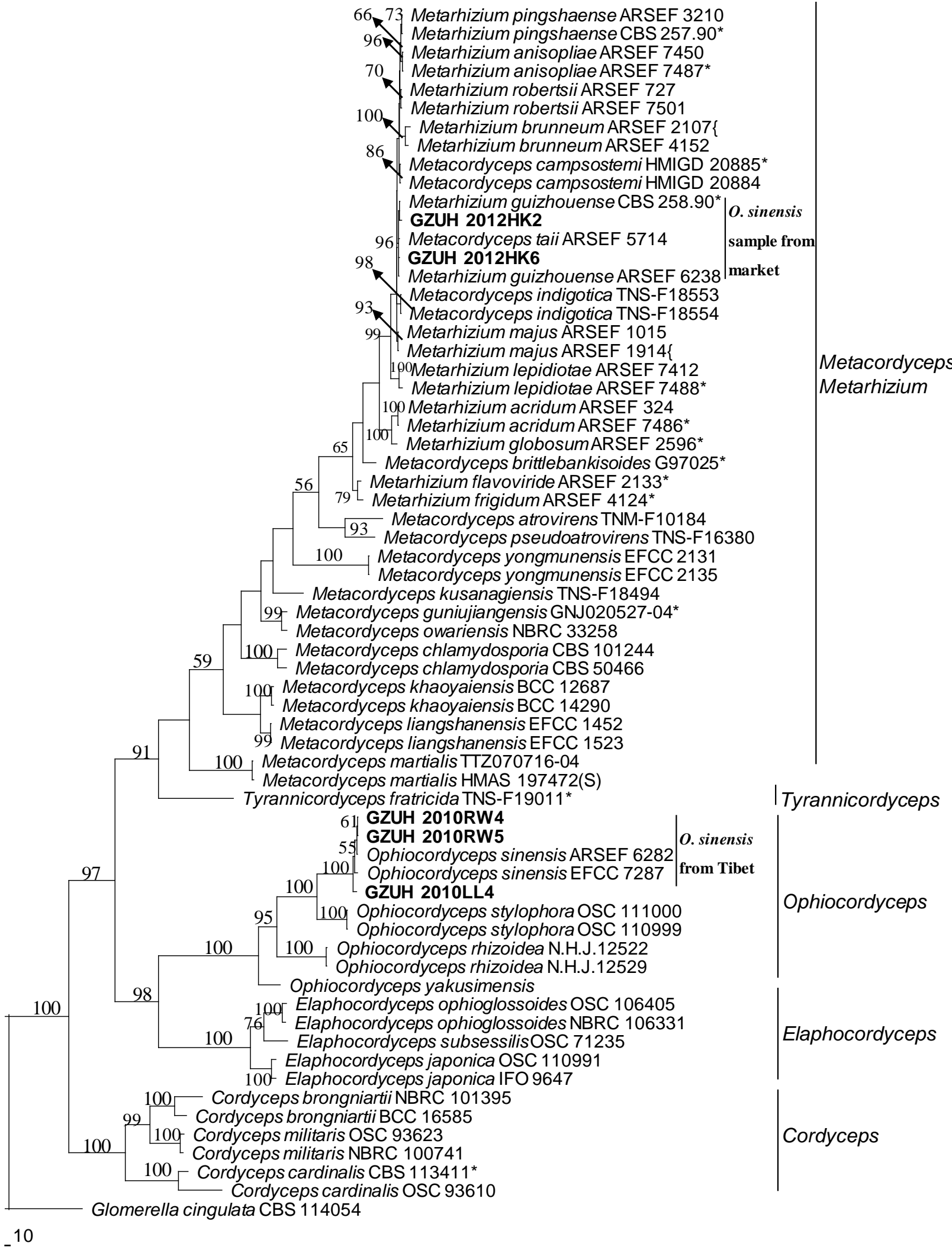

Figs 2 - Phylogenetic relationships among fake Ophiocordyceps sinensis and other related species based on combination of four genes (ITS, nrSSU, EF-1 $\alpha$, RPB1). Bootstraps values (1000 replicates) are indicated above the nodes. The tree is routed to Glomerella cingulata.

* Denotes an ex-type isolate. $\{$ Denotes an ex-epitype isolate. 

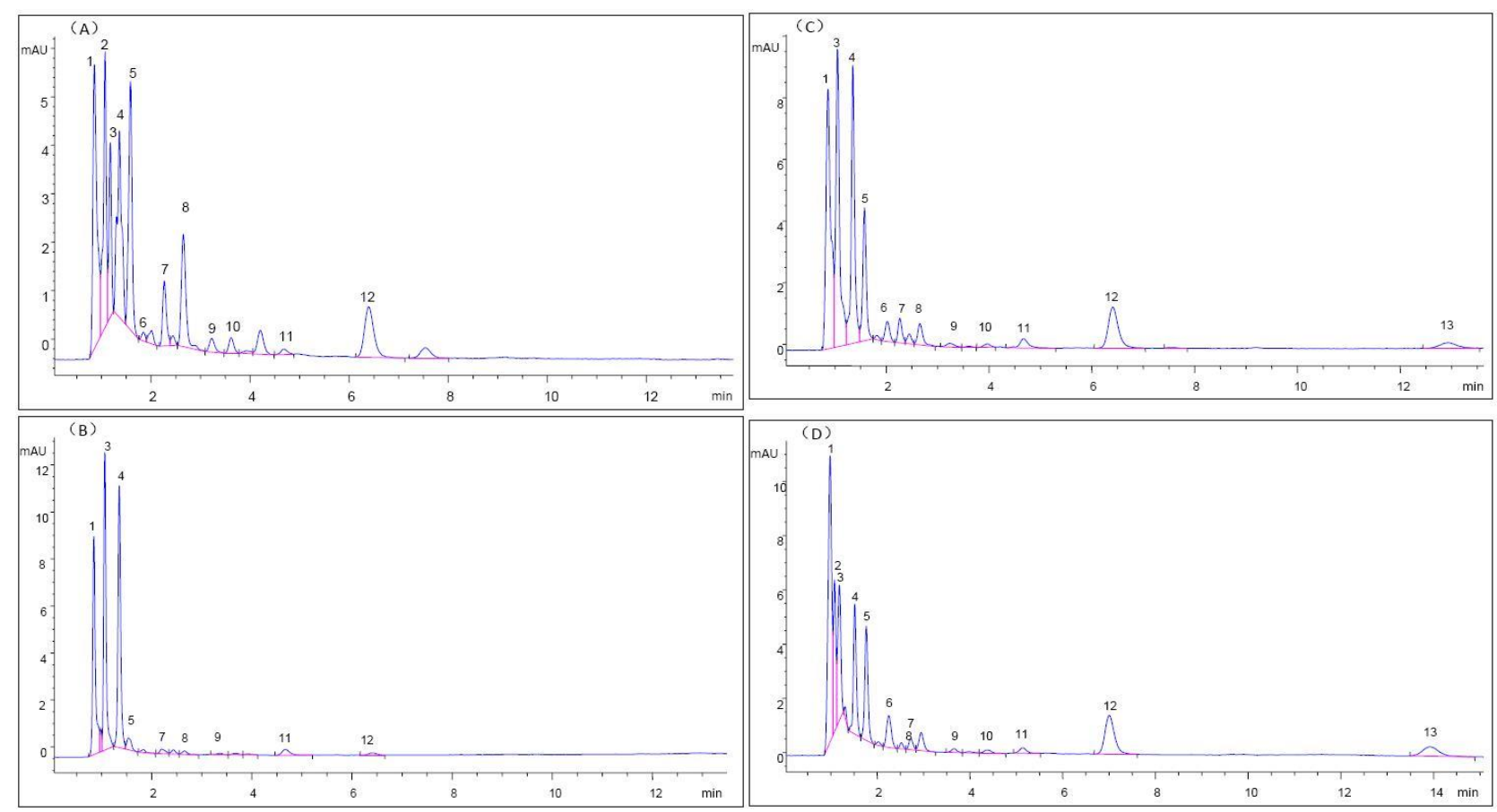

Fig. 3 - HPLC chromatograms of different simples. (A) Host of fake Ophiocordyceps sinensis; (B) Fruiting-body of fake $O$. sinensis; (C) Host of wild $O$. sinensis; (D) Fruiting-body of wild $O$. sinensis. 1: cytosine; 2: uracil; 3: guanine; 4: cytidine; 5: hypoxanthin; 6: adenine; 7: uridine; 8: thymine; 9: inosine; 10: guanosine; 11: thymidine; 12: adenosine; 13: undetermined.

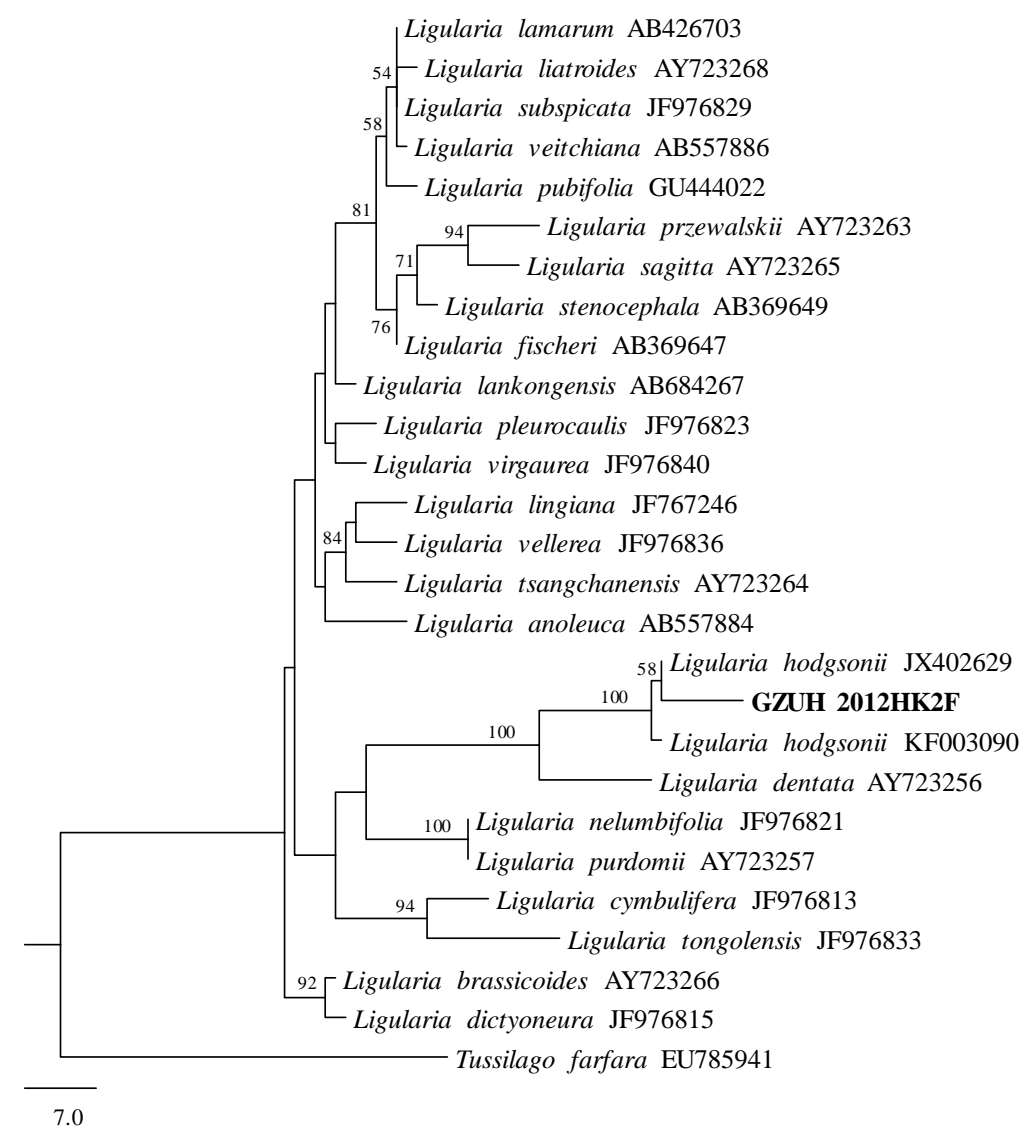

Fig. 4 - Phylogram generated from parsimony analysis based on ITS sequenced data of Ligularia. Parsimony bootstrap support values greater than $50 \%$ are indicated above the nodes. The sample from fake ones in this study is given in bold. The tree is rooted with Tussilago farfara. 
primer and probe designs for Ophiocordyceps sinensis in the future like as has been developed for some plant pathogenic fungi (Tao et al. 2013). As previous studies have indicated, an appropriate DNA barcode marker for fungal species identification is judged by two important criteria including the suitable intra- and interspecific variations and the high success rate of PCR amplification and sequencing (Hollingsworth 2009, Peng et al. 2011). In this study, we confirmed that the partial EF$1 \alpha$ and RPB1 are good DNA markers for Cordyceps sensu lato species as compared with ITS and nrSSU. ITS has been routinely used to study the phylogenetic relationships among different species of varies fungal groups and has also been regarded as the universal DNA barcode for the kingdom Fungi (Schoch et al. 2012, Nilsson et al. 2014), but does not work in many fungal groups (Maharachchikumbura et al. 2012, Hyde et al. 2014, Udayanga et al. 2014). In this study, we found that ITS and nrSSU have a high similarity among the tested species in Cordyceps sensu lato with some intra - and interspecific overlapping between species in the single gene tree.

The method for quality control is important to ensure authenticity and quality of Cordyceps species and products ( $\mathrm{Li}$ et al. 2006). In this study, chemical and multi-gene loci phylogeny are simultaneously used for identification and quality control of Ophiocordyceps sinensis; the methodology is quick and highly efficient. This is the first time to report fake $O$. sinensis made from Metacordyceps taii, however this method may become common for counterfeiting Ophiocordyceps sinensis in the future because of its remarkable similarity.

The strategies used for identification $O$. sinensis using combined sequenced data from the multi-gene loci could have a wide application in other TCM and in fungal biotechnology.

\section{Acknowledgements}

This work was supported by The National Natural Science Foundation of China (No.31460012 \& No.31611130034) and the Modernization of Traditional Chinese Medicine Program of Guizhou Province (No. 20125008).

\section{References}

Au D, Wang L, Yang D, Mok DK, Chan AS, Xu H. 2012 - Application of microscopy in authentication of valuable Chinese medicine I - Cordyceps sinensis, its counterfeits, and related products. Microscopy Research and Technique 75, 54-64.

Bischoff JF, Rehner SA, Humber RA. 2009 - A multilocus phylogeny of the Metarhizium anisopliae lineage. Mycologia 101, 512-530.

Cannon PF, Hywel-Jones NL, Maczey N, Norbu L, Tshitila, Samdup T, Lhendup P. 2009 - Steps towards sustainable harvest of Ophiocordyceps sinensis in Bhutan. Biodiversity \& Conservation 18, 2263-2281.

Castlebury LA, Rossman AY, Sung GH, Hyten AS, Spatafora JW. 2004 - Multigene phylogeny reveals new lineage for Stachybotrys chartarum, the indoor air fungus. Mycological Research 108, 864-872.

Chan WH, Ling KH, Chiu SW, Shaw PC, But PPH. 2011 - Molecular analyses of Cordyceps gunnii in China. Journal of Food and Drug Analysis 19, 18-25.

Cunningham CW. 1997 - Can three incongruence tests predict when data should be combined? Molecular Biology and Evolution 14, 733-740.

De Silva DD, Rapior S, Fons F, Bahkali AH, Hyde KD. 2012a - Medicinal mushrooms in supportive cancer therapies: an approach to anti-cancer effects and putative mechanisms of action. Fungal Diversity 55, 1-35.

De Silva DD, Rapior S, Hyde KD, Bahkali AH. 2012b - Medicinal mushrooms in prevention and control of diabetes mellitus. Fungal Diversity 56, 1-29.

De Silva DD, Rapior S, Sudarman E, Stadler M, Xu J, Alias SA, Hyde KD. 2013 - Bioactive metabolites from macrofungi: ethnopharmacology, biological activities and chemistry. Fungal Diversity 62, 1-40. 
Devkota S. 2000 - Ophicordyceps sinensis (Yarsagumba) from Nepal Himalaya: status, threats and management strategies. In: Zhang P, Hao W (Ed.), Cordyceps Resources and Environment. Grassland Supervision Center by the Ministry of Agriculture, People's Republic of China. 91-108.

Farris JS, Källersjö M, Kluge AG, Bult C. 1994 - Testing significance of incongruence. Cladistics 10, 315-319.

Felsenstein J. 1985 - Confidence Limitson Phylogenies: An Approach using the Bootstrap. Evolution 39, 783-791.

Gong YX, Li SP, Li P, Liu JJ, Wang YT. 2004 - Simultaneous determination of six main nucleosides and bases in natural and cultured Cordyceps by capillary electrophoresis. Journal Of Chromatography A 1055, 215-221.

Hirota H, Horiguchi Y, Kawaii S, Kuroda C, Hanai R, Gong X. 2012 - Four new bisabolane-type sesquiterpenes from Ligularia lankongensis. Natural Product Communications 7, 451-454.

Hollingsworth PM, Forrest LL, Spouge JL, Hajibabaei M, Ratnasingham S, van der Bank M, Chase MW, Cowan RS, Erickson DL, Fazekas AJ, Graham SW, James KE, Kim KJ, Kress WJ, Schneider H, van AlphenStahl J, Barrett SC, van den Berg C, Bogarin D, Burgess KS, Cameron KM, Carine M, Chacón J, Clark A, Clarkson JJ, Conrad F, Devey DS, Ford CS, Hedderson TA, Hollingsworth ML, Husband BC, Kelly LJ, Kesanakurti PR, Kim JS, Kim YD, Lahaye R, Lee HL, Long DG, Madriñán S, Maurin O, Meusnier I, Newmaster SG, Park CW, Percy DM, Petersen G, Richardson JE, Salazar GA, Savolainen V, Seberg O, Wilkinson MJ, Yi DK, Little DP. 2009 - A DNA barcode for land plants. Proceedings of the National Academy of Sciences 106, 12794-12797.

Hseu RS, Chen CS. Gene sequence and method for distinguishing Cordyceps sinensis. US Patent 6271003, 2001.

Hu K, Fang DS, Tang YP, Jia JJ, Li M. 2008 - Comparison of Cordyceps Sinensis and solid fermentation of Cordyceps Militaris by TLC. China Pharmacy 19, 1180-1182.

Hyde KD, Nilsson RH, Alias SA, Ariyawansa HA, Blair JE, Cai L, de Cock AWAM, Dissanayake AJ, Glockling SL, Goonasekara ID, Gorczak M, Hahn M, Jayawardena RS, van Kan JAL, Laurence MH, Lévesque CA, Li X, Liu JK, Maharachchikumbura SSN, Manamgoda DS, Martin FN, McKenzie EHC, McTaggart AR, Mortimer PE, Nair PVR, Pawlowska J, Rintoul TL, Shivas RG, Spies CFF, Summerell BA, Taylor PWJ, Terhem RB, Udayanga H, Vaghefi N, Walther G, Wilk M, Wrzosek M, Xu JC, Yan JY, Zhou N. 2014 - One stop shop: backbones trees for important phytopathogenic genera: I (2014). Fungal Diversity 67, 21125.

Jiang Y, Yao Y. 2003 - Anamorphic fungi related to Cordyceps sinensis. Mycosystema 22, 161176.

Kepler RM, Sung GH, Ban S, Nakagiri A, Chen MJ, Huang B, Li ZZ, Spatafora JW. 2012 - New teleomorph combinations in the entomopathogenic genus Metacordyceps. Mycologia 104, 182-197.

Kuo YC, Tsai WJ, Shiao MS, Chen CF, Lin CY. 1996 - Cordyceps sinensis as an immunomodulatory agent. American Journal of Chinese Medicine 24, 111-125.

Lei W, Chen H, Zhang GR, Li SS, Peng QY, Zhong X and Liu X. 2011 - Molecular identification and food source inference of constructive plants, native to the Ophiocordyceps sinensis habitat. African Journal of Biotechnology 10, 159-167.

Li CR, Huang B, Fan MZ, Lin YR, Li ZZ. 2010 - Metacordyceps guniujiangensis and its Metarhizium anamorph: a new pathogen on cicada nymphs. Mycotaxon 111, 221-231.

Li DS, Liu ZX, Zhang Y, Zhang CK. 2002 - Contrast analysis of mainly chemical ingredients of Cordyceps menshanensis and Cordyceps sinensis. Edible Fungi of China 21, 35-37.

Li DZ, Gao LM, Li HT, Wang H, Ge XJ, Liu JQ, Chen ZD, Zhou SL, Chen SL, Yang JB, Fu CX, Zeng CX, Yan HF, Zhu YJ, Sun YS, Chen SY, Zhao L, Wang K, Yang T, Duan GW. 2011 Comparative analysis of a large dataset indicates that internal transcribed spacer (ITS) should 
be incorporated into the core barcode for seed plants. Proceedings of the National Academy of Sciences of the United States of America 108, 19641-19646.

Li J, Feng CQ, NI XM, Zhang WS. 2008 - Determination of nucleosides of natural Cordyceps sinensis in Qinghai province by capillary electrophoresis. Chinese Pharmaceutical Journal 43, 1105-1107.

Li S, Yang F, Tsim KWK. 2006 - Quality control of Cordyceps sinensis, a valued traditional Chinese medicine. Journal of Pharmaceutical and Biomedical Analysis 41, 1571-1584.

Li SP, Li P, Lai C, Gong YX, Kan KK, Dong TT, Tsim KW, Wang YT. 2004a - Simultaneous determination of ergosterol, nucleosides and their bases from natural and cultured Cordyceps by pressurised liquid extraction and high-performance liquid chromatography. Journal of Chromatography A 1036, 239-243.

Li SP, Song ZH, Dong TTX, Ji ZN, Lo CK, Zhu SQ, Tsim KWK. 2004b - Distinction of watersoluble constituents between natural and cultured Cordyceps by capillary electrophoresis. Phytomedicine 11, 684-690.

Li XQ, Wang Y, Bao TT. 1999 - RP-HPLC determination of adenosine in fermented Cordyceps. China journal of Chinese materia medica 24, 12-14, 62.

Liu JQ, Wang YJ, Wang AL, Hideaki O, Abbott RJ. 2006 - Radiation and diversification within the Ligularia-Cremanthodium-Parasenecio complex (asteraceae) triggered by uplift of the Qinghai-Tibetan plateau. Molecular Phylogenetics and Evolution 38, 31-49.

Liu ZY, Liang ZQ, Liu AY, Yao YJ, Hyde KD, Yu ZN. 2002 - Molecular evidence for teleomorph-anamorph connections in Cordyceps based on ITS-5.8S rDNA sequences. Mycological Research 106, 1100-1108.

Ma Y, Wang Y, Yang GZ, Yu RM. 2008 - Determination of nucleosides in Cordyceps sinensis preparation by dual-wavelength TLC-scanning. China Pharmacy 19, 2375-2377.

Maharachchikumbura SSN, Guo LD, Cai L, Chukeatirote E, Wu WP, Sun X, Crous PW, Bhat DJ, McKenzie EHC, Bahkali AH, Hyde KD. 2012 - A multi-locus backbone tree for Pestalotiopsis, with a polyphasic characterization of 14 new species. Fungal Diversity 56, 95-129.

Mortimer PE, Karunarathna SC, Li Q, Gui H, Yang X, Yang X, He J, Ye L, Guo J, Li H. 2012 Prized edible Asian mushrooms: ecology, conservation and sustainability. Fungal Diversity $56,31-47$.

Nagano H, Kanda M, Yamada H, Hanai R, Gong X, Kuroda C. 2010 - Chemical and genetic study of Ligularia anoleuca and L.veitchiana in Yunnan and Sichuan provinces of China. Helvetica Chimica Acta 93, 1945-1952.

Nikoh N, Fukatsu T. 2000 - Interkingdom host jumping underground: phylogenetic analysis of entomoparasitic fungi of the genus Cordyceps. Molecular Biology and Evolution 17, 629-638.

Nilsson RH, Hyde KD, Pawłowska J, Ryberg M, Tedersoo L, Aas AB, Alias SA, Alves A, Anderson CL, Antonelli A, Arnold AE, Bahnmann B, Bahram M, Bengtsson-Palme J, Berlin A, Branco S, Chomnunti P, Dissanayake A, Drenkhan R, Friberg H, Frøslev TG, Halwachs B, Hartmann M, Henricot B, Jayawardena R, Jumpponen A, Kauserud H, Koskela S, Kulik T, Liimatainen K, Lindahl BD, Lindner D, Liu JK, Maharachchikumbura S, Manamgoda D, Martinsson S, Neves MA, Niskanen T, Nylinder S, Pereira OL, Pinho DB, Porter TM, Queloz V, Riit T, Sánchez-García M, de Sousa F, Stefańczyk E, Tadych M, Takamatsu S, Tian Q, Udayanga D, Unterseher M, Wang Z, Wikee S, Yan JY, Larsson E, Larsson KH, Kõljalg U, Abarenkov K. 2014 - Improving ITS sequence data for identification of plant pathogenic fungi. Fungal Diversity 67, 11-19.

Nomura N, Takaso T, Peng C, Kono Y, Oginuma K, Mitsui Y, Setoguchi H. 2010 - Molecular phylogeny and habitat diversification of the genus Farfugium (asteraceae) based on nuclear rDNA and plastid DNA. Annals of Botany 106, 467-482.

Page RDM. 1996 - TreeView: an application to display phylogenetic trees on personal computers. Computer Applications in the Biosciences Cabios 12, 357-358. 
Peng Z, Jing L, Zhuang WY, Liu XZ, Bing W. 2011 - Erratum to:DNA barcoding of the fungal genus Neonectria and the discovery of two new species. Science China Life Sciences 54, 664-674.

Saito Y, Hattori M, Iwamoto Y, Takashima Y, Mihara K, Sasaki Y, Fujiwara M, Sakaoku M, Shimizu A, Chao X, Kuroda C, Gong X, Hanai R, Tori M. 2011 - Overlapping chemical and genetic diversity in Ligularia lamarum and Ligularia subspicata. isolation of ten new eremophilanes and a new seco-bakkane compound. Tetrahedron 67, 2220-2231.

Schneider S, Rehner SA, Widmer F, Enkerli J. 2011 - A PCR-based tool for cultivationindependent detection and quantification of Metarhizium clade 1. Journal of Invertebrate Pathology 108, 106-114.

Schoch CL, Seifert KA, Huhndorf S, Robert V, Spouge JL, Levesque CA, Chen W, Fungal Barcoding Consortium. 2012 - Nuclear ribosomal internal transcribed spacer (ITS) region as a universal DNA barcode marker for Fungi. Proceedings of the National Academy of Science 109, 6241-6246.

Shrestha UB, Bawa KS. 2013 - Trade, harvest, and conservation of caterpillar fungus (Ophiocordyceps sinensis) in the Himalayas. Biological Conservation 159, 514-520.

Shrestha UB. 2012 - Asian medicine: A fungus in decline. Nature 482 (7383):35.

Singh N, Rakshita P, Kathait AS, Rautela D, Dubey A. 2010 - Collection of Cordyceps sinensis (Berk.) Sacc. in the interior villages of Chamoli District in Garhwal Himalaya (Uttarakhand) and its social impacts. Journal of American Science 6, 5-9.

Sun YQ, Sun GX, Jin Y. 2008 - Quality control of traditional Chinese medicines by the capillary electrophoresis fingerprint and capillary electrophoresis-mass spectrometry. Chinese Journal of Chromatography 26, 160-165.

Sung GH, Sung JM, Hywel-Jones NL, Spatafora JW. 2007 - A multi-gene phylogeny of Clavicipitaceae (Ascomycota, Fungi): Identification of localized incongruence using a combinational bootstrap approach. Molecular Phylogenetics and Evolution 44, 1204-1223.

Swofford DL. 2002 - PAUP*: phylogenetic analysis using parsimony (*and other methods), version 4.0b10. Sinauer Associates, Sunderland.

Tamura K, Peterson D, Peterson N, Stecher G, Nei M, Kumar S. 2011 - MEGA5: molecular evolutionary genetics analysis using maximum likelihood, evolutionary distance, and maximum parsimony methods. Molecular Biology and Evolution 28, 2731-2739.

Tao G, Hyde KD, Cai L. 2013 - Species-specific real-time PCR detection of Colletotrichum kahawae. Journal of Applied Microbiology 114, 828-835.

The State Pharmacopoeia Committee of People Republic of China. 2010 - Pharmacopoeia of the People's Republic of China. Part I. Beijing: Chemical Industry Publishing House, 106, 352.

Tuli HS, Sandhu SS, Sharma AK. 2013 - Pharmacological and therapeutic potential of Cordyceps with special reference to Cordycepin. 3 Biotech 4, 1-12.

Udayanga D, Castlebury LA, Rossman AY, Chukeatirote E, Hyde KD. 2014 - Insights into the genus Diaporthe: phylogenetic species delimitation in the D. eres species complex. Fungal Diversity 67, 203-229.

Wen TC, Zhu RC, Kang JC, Huang MH, Tan DB, Ariyawansha H, Hyde KD, Liu H. 2013 Ophiocordyceps xuefengensis sp. nov. from larvae of Phassus nodus (Hepialidae) in Hunan Province, southern China. Phytotaxa 123, 41-50.

Weng RA, Li SH. 2008 - Identification of Cordyceps and its adulterants Cordyceps militaris using ITS sequencing. Journal of Hunan Normal University (Medical Science) 5, 42-45.

White TJ, Bruns T, Lee S, Taylor JW. 1990 - Amplification and direct sequencing of fungal ribosomal RNA genes for phylogenetics. PCR Protocols A Guide to Methods and Applications, 315-322.

Winkler D. 2009 - Caterpillar fungus (Ophiocordyceps sinensis) production and sustainability on the Tibetan Plateau and in the Himalayas. Asian Medicine 5, 291-316. 
Wu TN, Yang KC, Wang CM, Lai JS, Ko KN, Chang PY, Liou SH. 1996 - Lead poisoning caused by contaminated Cordyceps, a Chinese herbal medicine: two case reports. Science of the Total Environment 182, 193-195.

Xiang L, Song JY, Xin TY, Zhu YJ, Shi LC, Xu XL, Pang XH, Yao H, Li WJ, Chen SL. 2013 DNA barcoding the commercial Chinese caterpillar fungus. FEMS Microbiology Letters 347, 156-162.

Yu JJ, Kuroda C, Gong X. 2011 - Natural hybridization and introgression in sympatric Ligularia species (asteraceae, senecioneae). Journal of Systematics and Evolution 49, 438-448.

Yue K, Ye M, Zhou Z, Sun W, Lin X. 2013 - The genus Cordyceps: a chemical and pharmacological review. Journal of Pharmacy and Pharmacology 65, 474-493.

Zhang WM, Hu B, Zhong H, Chen YQ, Tao MH. 2005 - Study on the anamorph of Cordyceps campsosterna. Proceedings of the First Symposium on Development of China's Medicinal Fungi Industry 81-82.

Zhou XW, Gong ZH, Su Y, Lin J, Tang KX. 2009 - Cordyceps fungi: natural products, pharmacological functions and developmental products. Journal of Pharmacy and Pharmacology 61, 279-291.

Zhu JS, Halpern GM, Jones K. 1998 - The scientific rediscovery of a precious ancient Chinese herbal regimen: Cordyceps sinensis: part II. Journal of Alternative and Complementary Medicine 4, 429-457. 